\title{
Fire Detection Using CNN \& SVM
}

\author{
Saranya M, Tamilselvan Arunachalam, Nijanthan N \\ \{*msa.ice@psgtech.ac.in, tamilselvanpsg16u253@gmail.com, nijannija99@gmail.com\} \\ Assisant Professor,Dept. of I\&CE,PSG College of Technology,Tamilnadu,Coimbatore,India ${ }^{1}$ Dept. of I\&CE, \\ PSG College of Technology, Coimbatore, India ${ }^{2}$ Dept. of I\&CE, PSG College of Technology, Coimbatore, \\ India $^{3}$
}

\begin{abstract}
Fire detection in outdoor which can't accomplished by sensor. The fire will endanger property and life of humans. An early caution is very important to prevent the damage that could occur to property and life of human. In indoor, there is possibility of false alarm detection due to change that occurs in the environment. So the best alternative solution to avoid the false alarm is the Image processing based fire detection. Using MATLAB software as a tool in image processing, we can identify whether there is fire or not. This paper proposes a classifying algorithm for identification of the input image as fire or non-fire case based on some statistical features obtained from the images. The extracted features from the input image are provided as input to the SVM to classify them. In addition to SVM, we are using CNN for further improvement because CNN is pre-trained model.
\end{abstract}

Keywords-Features, SVM, Learner, Filter, Pooling, CNN, Alexnet

\section{Introduction}

Regular monitoring of the environment and urban areas with fire detection system is very essential nowadays, to avoid the occurrence of damage to property and people's lives. The conventional and traditional way to detect fires is by using a sensor, but some sensors have a proximity range and cannot cover a large area. Rapid advancement of digital Technology, digital camera technology and video processing techniques have advanced even further. As a result, it was possible to replace traditional fire detection technologies with a new fire detection system that relied heavily on image processing techniques. From incomplete statistics surveyed in 2016, there were around 312,000 fire occurrences in the country. That resulted in the deaths of 1,582 people, injuries to 1,065 people, and the loss of property of $\$ 3.72$ billion [1,2]. Many large-scale fire mishaps occurred in 2019 all across the world. Notre Dame (France), Liangshan (China) and the meadow fire (Russia) are only a few of the most well-known incidents. These accidents resulted in significant loss of life and property.

Therefore, the fire detection system is necessary for the safety of people and wildlife. Currently, much research is carried out in this field to provide safety to humans in advance. Few outstanding research activities include classification based on fire pixels. Both grayscale and colour video material might be considered. Color video footage typically use rules-based fire pixel classification. Chen, et al. [3] developed a rule system for categorising fire pixels based on the raw R, G, and B colour information. Kumarguru Poobalan et al. [4] used the edge detection method to build fire detection systems. [5] Turgay Celik et al. Using the backdrop subtraction methodology to identify moving pixels, a model-based color-based method to detect fiery pixels was developed. By assessing the dynamics of the regions and triggering the fire alarm in relation to the dynamics of the region, the background model was updated to recognise regions of moving fire pixels. The YCbCr colour space was utilised by Mubarak A.I. Mahmoud Honge Ren et al. [6] for fire detection because it successfully differentiates luminance from chrominance and can thus separate pixels from the centre of fire at high temperatures. Because the fire in the middle high-temperature region is white, this is the case. YCBCR is used by Ti Nguyen et al. [7] to extract colour and motion from video footage for fire detection. Using image processing techniques and classification algorithms such as SVM and CNN, this paper provides a method for detecting fires. 


\section{System Description}

\section{A. $\quad$ Proposed Solution}

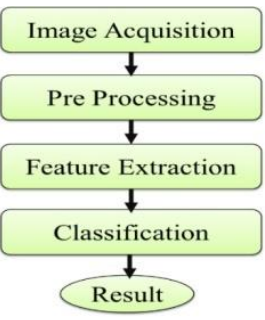

Fig 1. Block Diagram

Obtaining image of the place from the data set and applying various pre- processing technique, so that the distinguishing features can be extracted. These extracted features are then fed in to a classifier for classifying the fire image from the non-fire image. Fig .1 depicts the proposed algorithm. Data set is considered to provide the offline data.

B.System Description

In technical processing MATLAB is the high performance language. It includes visualize effect, computation, programming for problems and provide solution in mathematical terms. General uses include:

- Mathematical computation

- Development of algorithm

- Simulation and Prototype

- Data Analysis

- Engineering graphics

- Building of Graphical User Interface

Matlab is an interactive platform and array is the basic element which does not require dimensioning. Vector formulations, matrix and technical computing problems can be solved in the MATLAB and for writing program it would take a fraction of time in non- interactive programming language like $\mathrm{C}$ or Fortran.

MATLAB is in use for many years and it provide more user friendly functionalities to multiple domain users. It is the instructional based standard type of tool used for the purpose of introductory in universities and maths, engineering and science. For high productivity research, MATLAB is main tool in the industry for development and analysis.

MATLAB features has toolboxes in it. It allows the user to learn and apply the special technology. By using the toolboxes collection of the M-files in MATLAB function can extend its functionalities to solve a particular problems. Control System, Signal processing, simulation, fuzzy logic, simulation, neural networks are the areas will toolboxes available.

\section{C.Support Vector Machine}

One of the central classification algorithms is a SVM classifier. It is a type of supervised learning (SL) system. SL have a dataset provided with both features and class labels. Result assessment can be performed using classification learner. Classification learner assists the user to select the optimum model for your data by performing model comparisons and assessment in terms of ROC curves and confusion matrices Once assessed, the trained models to classify the latest data are exported to the MATLAB workspace, thereby the predictions on the current or future data. It also allows us to transfer the models such as processing signals, analyzing data and computer vision. In MATLAB 2018b, SVM is available as application which is easy to handle through user interface. 
D.Convolution Neural Network

CNN is a type of Deep Learning (DL) technique that can be used for picture categorization and analysis. In CNN, before train the neural network, filter the image take place. Filter is set of multiplier. Pooling is process of reducing information but feature is still maintain. There are lot of convolution layer where randomly initialized filter will filter input image. Matching performed by Neural Network. Over time, perfect match will learned which is called feature extraction.

\section{E.Alexnet}

It's a pre-trained CNN that was trained with about 1.2 million images from the ImageNet Dataset. It has eight layers, each with its own set of weights; of the eight layers, five are convolutional and three are completely connected. This network accepts a 227 by 227 picture as input. In MATLAB 2018b, AlextNet is not a default app. The command net=alexanet must be used to activate it. When the programme above is run, it returns an Alexnet network that has been trained on the ImageNet data set.

\section{Feature Extraction}

The practise of decreasing the number of resources required to accurately describe a large quantity of data is known as feature extraction. The vast number of variables involved is one of the most difficult aspects of conducting extensive data analysis. When dealing with a large number of variables, you'll either require a lot of memory and processing power, or a classification algorithm that overfits the training sample and does not generalise well to new samples. Feature extraction is a broad term that relates to methods for combining variables to overcome problems while still accurately characterising data.

\section{A. Mean}

The mean here is the average intensities present in the image at different planes.

$$
\mu=\sum_{i=1}^{i=n I_{i}} n
$$

where $\mathrm{I}$ and $\mathrm{n}$ is the intensity and no of pixels respectively.

B. Standard Deviation

Standard Deviation quantifies the variation amount or dispersion of a set of data values.

$$
\sigma=\left(\sum_{i=1}^{n} \frac{(1-\mu)}{n}\right)
$$

\section{Entropy} One

Randomness in dataset can be measured using the Entropy. It characterizes the texture of the input image. of the feature that provides discriminatory changes in texture of the normal and non-fire image.

Entropy $=-(\operatorname{sum}(p \cdot * \log 2 p))$

where $\mathrm{p}$ is the probability of $i_{\text {th }}$ pixel in the image.

D. Gray Level Co-occurrence Matrix (GLCM)

Domain-specific qualities such as colour, texture, and shape are examples of first-order features. The Gray Level Co-occurrence Matrix aids in the extraction of statistical texture features of the second order. This GLCM aids in motion estimate based on input images. At a particular offset in the input image, a co-occurrence 
distribution matrix offers information about the distribution of co-occurring pixel values in terms of grayscale or colour values [8].

$$
\begin{aligned}
& C \Delta x, \Delta y(i, j)= \\
& \sum n x=1 \sum m y=1\{1, \text { if }(x, y)=i 0 \text { and, oth erwise }(x+\Delta x, y+\Delta y)=j
\end{aligned}
$$

$\Delta x, \Delta y$ is a position operator which is applied to the any pixel in an image. If input image has $\mathrm{P}$ different pixel value, then it will yield P X P matrix. The co-ocurrence matrix value at $(\mathrm{i}, \mathrm{j})$ is the number of times $i t h$ and $j$ th pixel repeated in the image. From the GLCM, the following features can be extracted.

1. Contrast: Over the entire image, it is the measure of the intensity contrast between a pixel and its neighbour.

$$
C=\sum i,(i-j) 2 p(i, j)
$$

2. Correlation: It is a measure of pixel to which extent it is correalted is to its neighbor over the whole image.

$$
C o=\sum i,(i-j) 2 p(i, j)
$$

3. Angular moment: It is the sum of squared elements in the GLCM.

$$
A M=\sum i,(p(i, j)) 2
$$

4. Homogeneity: Returns a value that measures the closeness data often incomplete, inconsistent, and/or lacking certain behaviors or trends and is likely contain many errors.

\section{Implementation}

First step is to start with collecting data,that is image acquisition. This involves collection of data from different sources and it is stored as offline. Such offline data base has 55 fire images and 55 non-fire images. In working with MATLAB, creating two folder for SVM and CNN process is good practice and helpful in accessing. For CNN, data are main source, so the image are resized to 227 x 227 as input size of AlexNet and stored as Datastore.

\section{A. Preprocessing}

Preprocessing involves processing of raw input data (i.e.) input image. It involves feature extraction. It transforms the raw input data into an understandable format. Here image resize and convert image to HSV where saturation region was obtained that shows in Fig 2 (sample). 


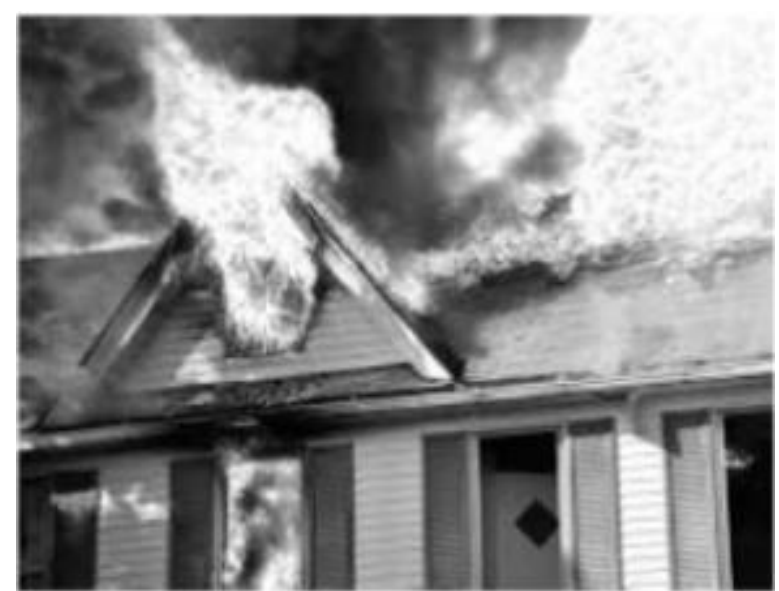

Fig 2. Pre-Processed Fire image

\section{B. Data Import}

After the feature extraction from image, the features and corresponding label are store in single table. Feature contain table should import in workspace for using in SVM application in MATLAB. There cross validation will take place shows in Fig 3.

\section{SVM selection}

SVM application has Linear SVM, fine Gaussian SVM, Medium Gaussian SVM, Quadratic, Cubic SVM, etc. We select one of SVMs and train the model based on best discriminative features. Using trial and error, we can select best trained Model. Selection process shown in Fig 4.

\section{Confusion Matrix}

A confusion matrix is a table that shows how well a classification model (or "classifier") performed on a set of test data for which the true values are known. It enables the visualisation of an algorithm's performance. A confusion matrix is a classification problem prediction outcome. The number of right and incorrect predictions is broken down by class and summarised with count values. A square matrix is a confusion matrix. The diagonal count, which is a valid forecast of the ranking, can be used to determine the ranking's correctness. A confusion matrix is a table that shows how well a classification model (or "classifier") performed on a set of test data for which the true values are known. It enables the visualisation of an algorithm's performance. A confusion matrix is a classification problem prediction outcome. The number of right and incorrect predictions is broken down by class and summarised with count values. A square matrix is a confusion matrix. The diagonal count, which is a valid forecast of the ranking, can be used to determine the ranking's correctness. Figure 5 shows the SVM classification confusion matrix. 


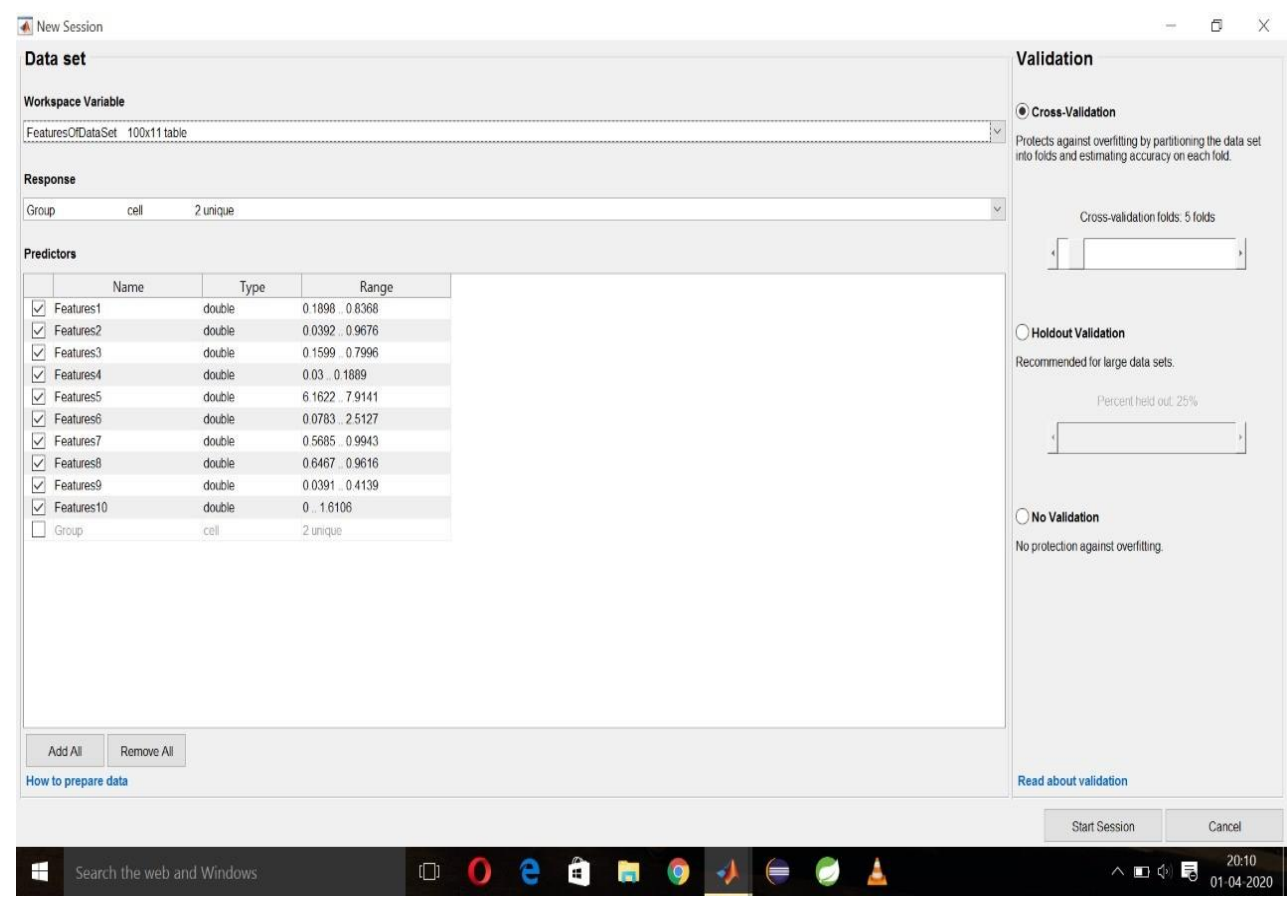

Fig 3. Data import and cross validation setup

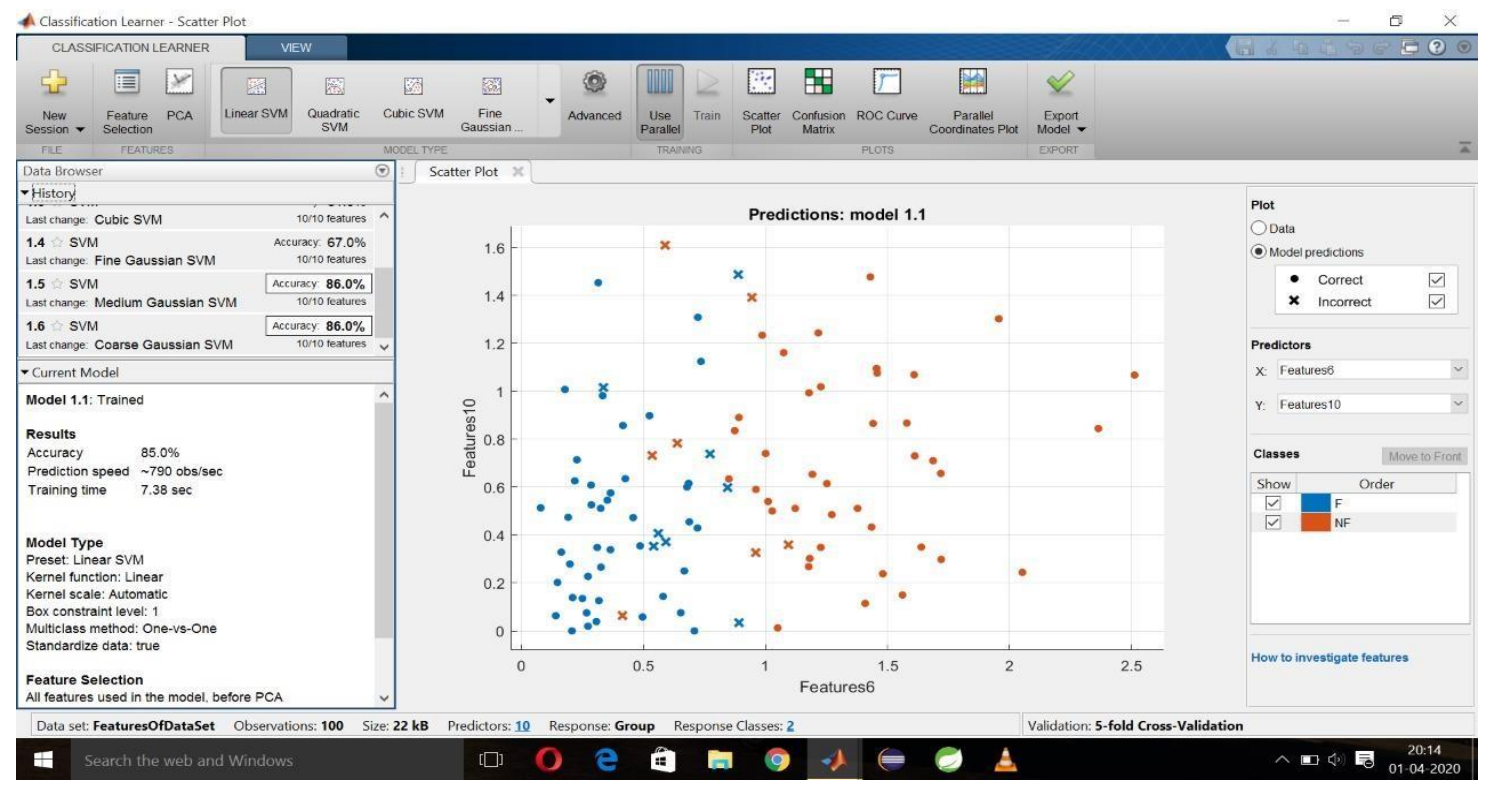

Fig 4. Selection of classification algorithm 


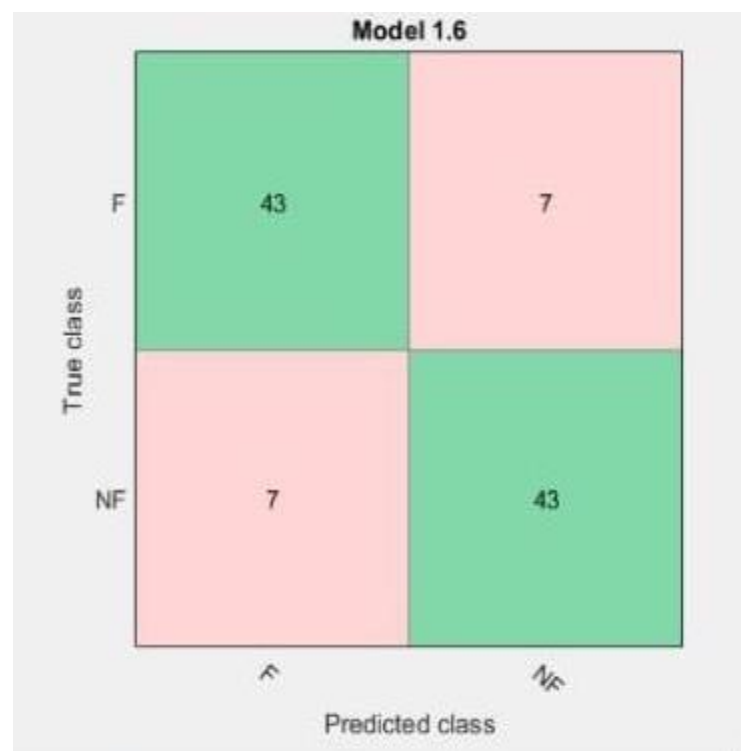

Fig 5. Confusion Matrix

Accuracy is computed using below equation

$$
\text { Accuracy }=\frac{\text { Sum of diagonal }}{100} * \text { Number of data (input) }
$$

\section{Result}

\section{A. Discriminatory features}

The features mentioned in chapter 2 was obtained for 100 images. Out of 100, 50 are fire containing images and 50 are non-fire images. There were some features of non-fire and fire containing images. Variance is one of the features changes, 0.65 is average discriminate line between fire and non-fire image. These are shown in Fig 6. In below figure, blue line indicate that they are fire images and the brown indicate that they are non-fire image. Fig 7 shows the one of the change between fire and non-fire image with entropy. Here the result shows that most of fire images are below the 7.5. In Fig 8 and Fig 9 blue line indicate that they are fire images and the brown indicate that they are non-fire image

The contrast between the fire and non-fire images, so consider as discriminatory change. This change is taken for the number of samples and shown in Fig 8. The correlation means how the data is related to itself. Fig 9 shows how the fire and the non-fire are how related to itself. Homogeneity means sameness that is how 50 samples of the fire images close to the each samples likewise for images these shown in Fig 9. In below figures, the blue line indicate that they are fire images and the brown indicate that they are nonfire image. Fig 10 shows that energy between the fire and non-fire images and all images have different energy value.

\section{B. SVM Classifier Result}

SVM stands for supervised machine learning and can be used to solve classification and regression problems. As we using MATLAB tool, there is a Classifier application which help us to implement. The features are extracted from preprocessed image and corresponding labels are given to the application with help user 
interface. The result from trained model through SVM is discussed below. Fig 11 shows the fire image and Fig 12 shows pre-processed image and Fig 13 shows that staturated image which is get from pre-processed image Fig 14 shows that result of SVM for fire image and SVM gives that the image has by showing the result as 'F'. From Fig 14, it clearly says that input image has fire and input image is from test image set, Fig 15 shows that result of SVM for non fire image and SVM gives that the image has by showing the result as 'NF'. From Fig 15, it clearly says that input image has no fire and input image is from test image set.

\section{C . Convolution Neural Network (CNN) Result}

For neural network approach we used alexnet which is a pretrained model.In MATLAB, we created a folder Datastore which contain both Fire and Non-Fire image folder which serves as source for dataset in addition to test folder which is useful to validate the model. The transfer learning approach starts with load the alexnet in MATLAB and 70\% dataset of both fire and non-fire image serves for training and $30 \%$ for test. Features are extracted from layer fc8 in alexnet and using error-correcting output codes (ECOC) model build a module to classify. Fig 16 shows that number input provided to classifier and these images are available in test folder. Fig 17 shows that result of neural network classifier. The time take by the end module developed in MATLAB $2018 \mathrm{~b}$ to detect the fire images is shown in Fig 18. More time consuming process is importing of alexnet which is 6.533s.

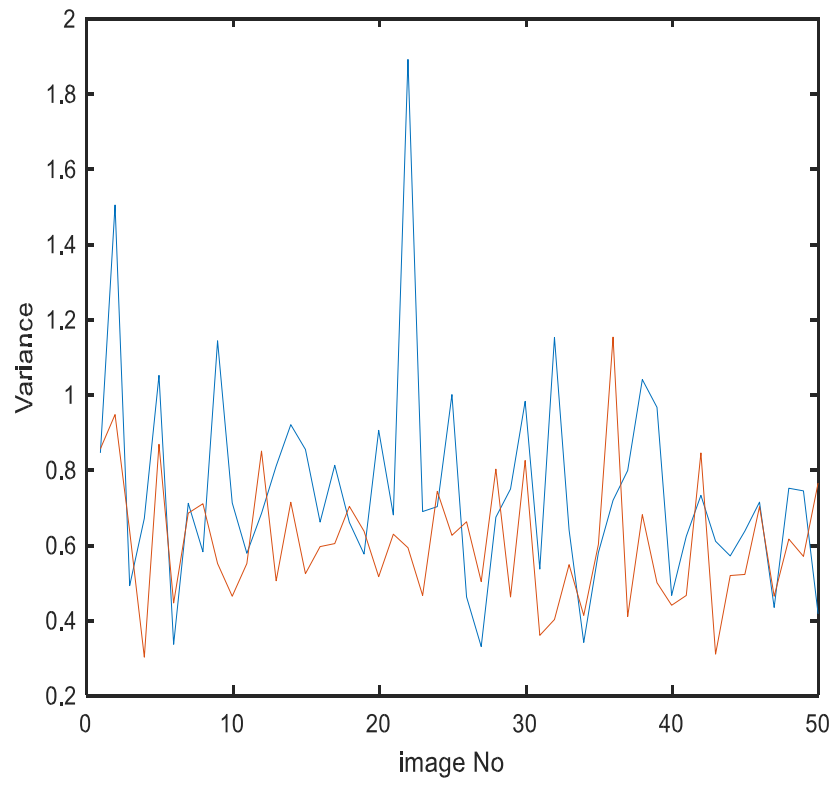

Fig 6 Variance 


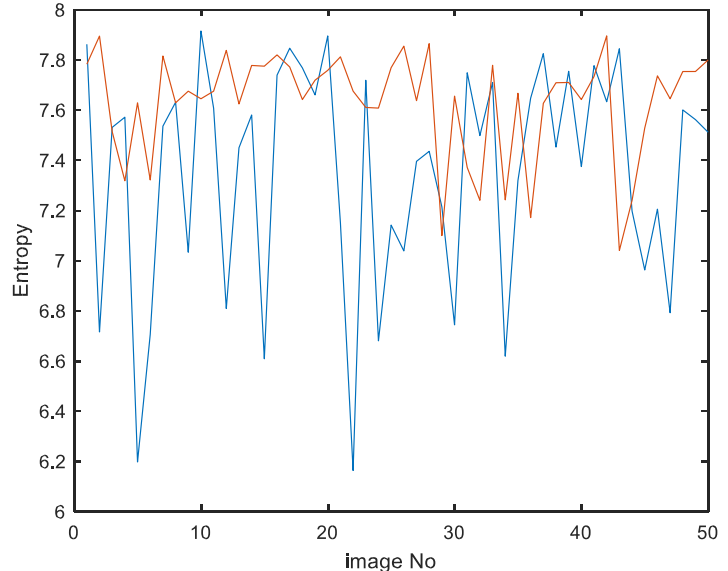

Fig. 7 Entropy

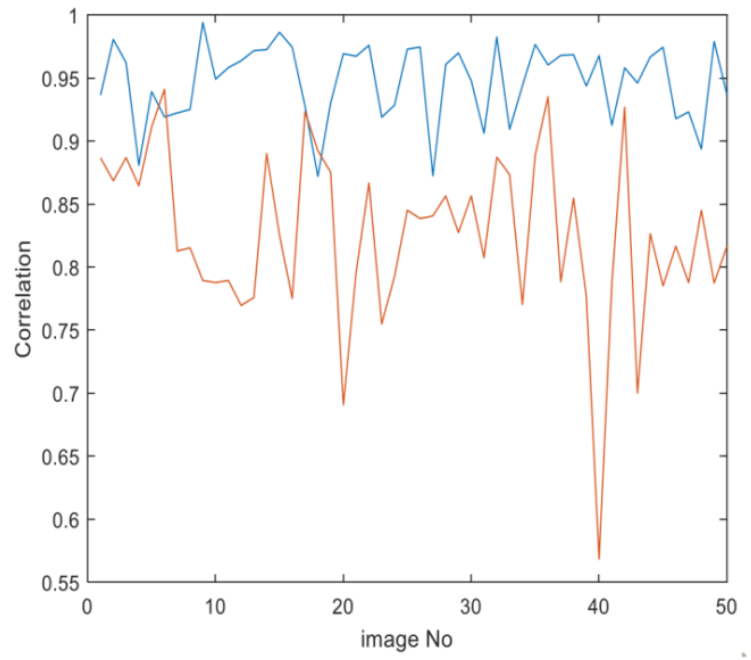

Fig 8 Correlation

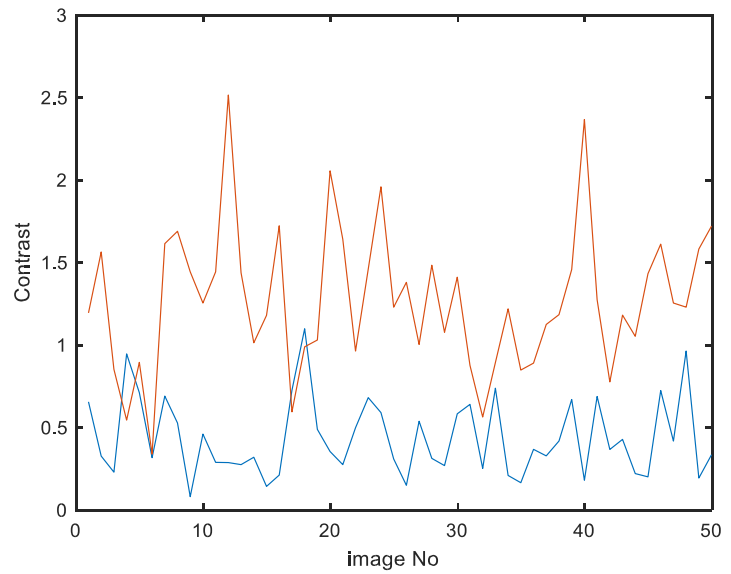

Fig 9 Homogeneity

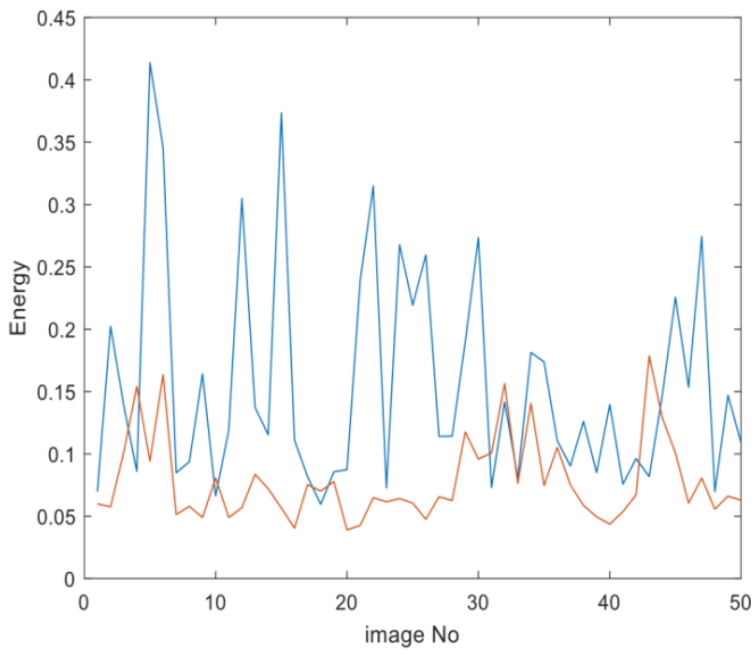

Fig 10 Energy 


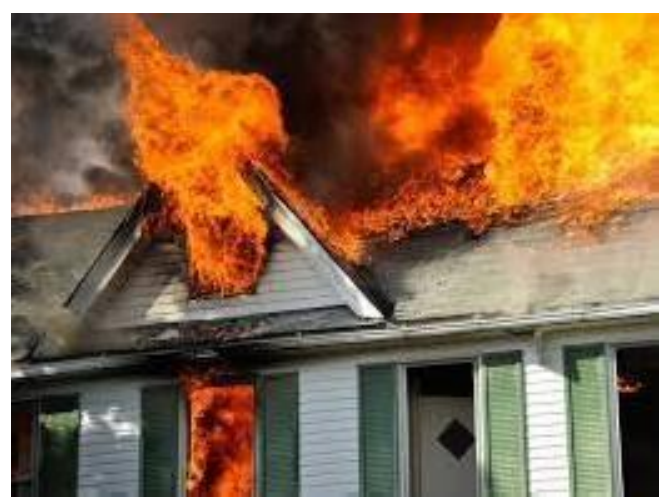

Fig.11 Fire image

Fig. 12 Pre-processed

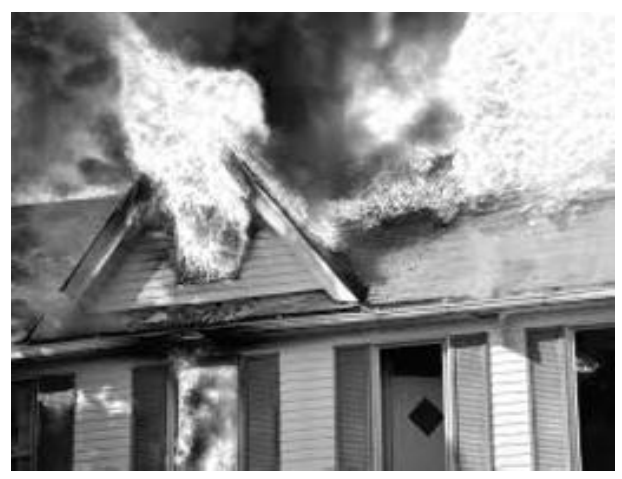

Fig 13 Saturated image 


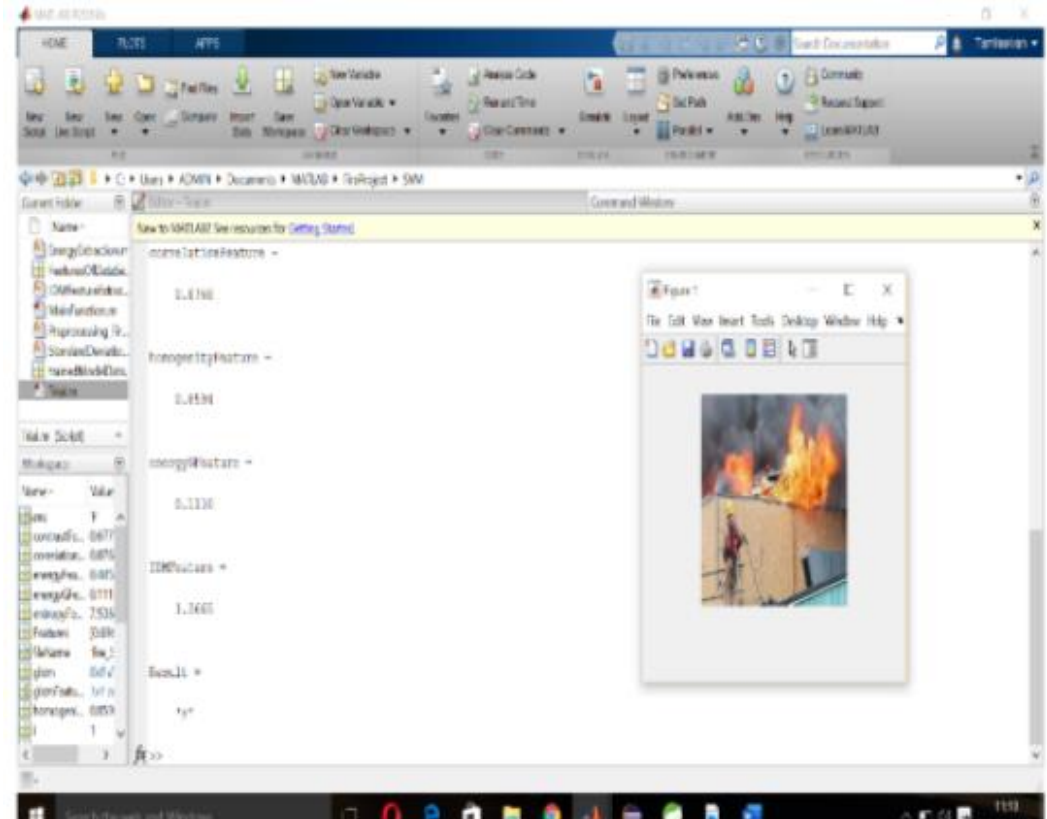

Fig 14 Result of SVM for fire image

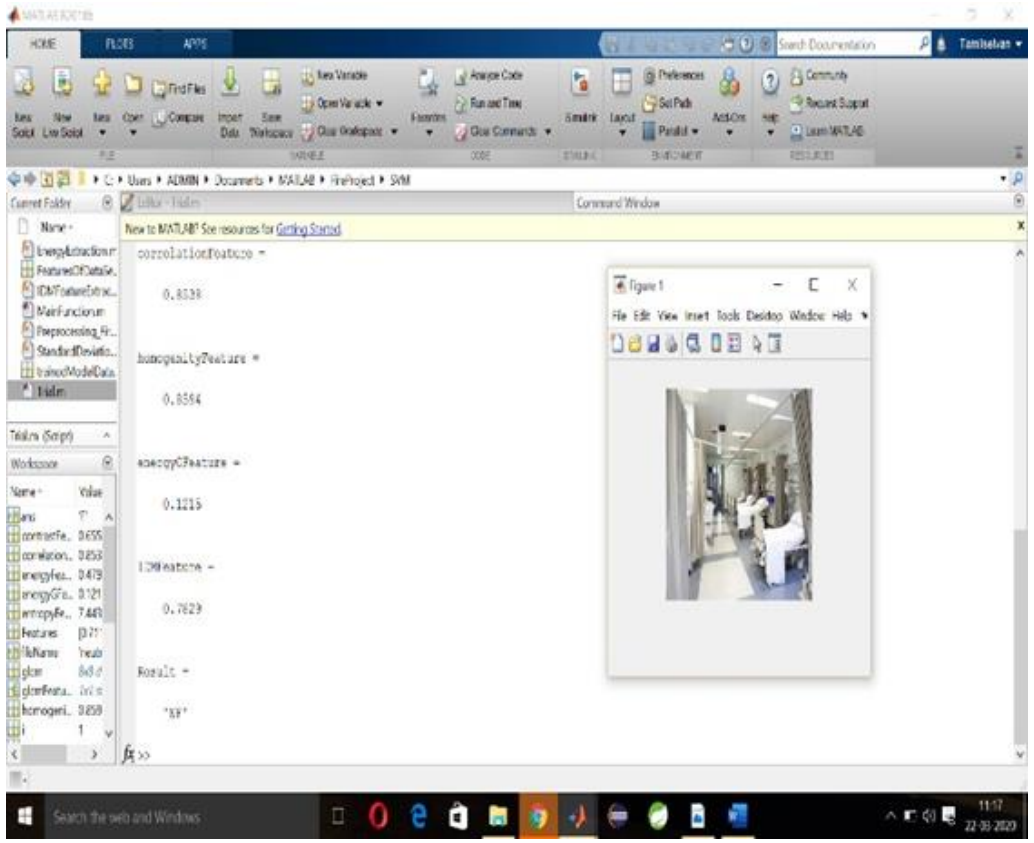

Fig 15 Result of SVM for non fire image 


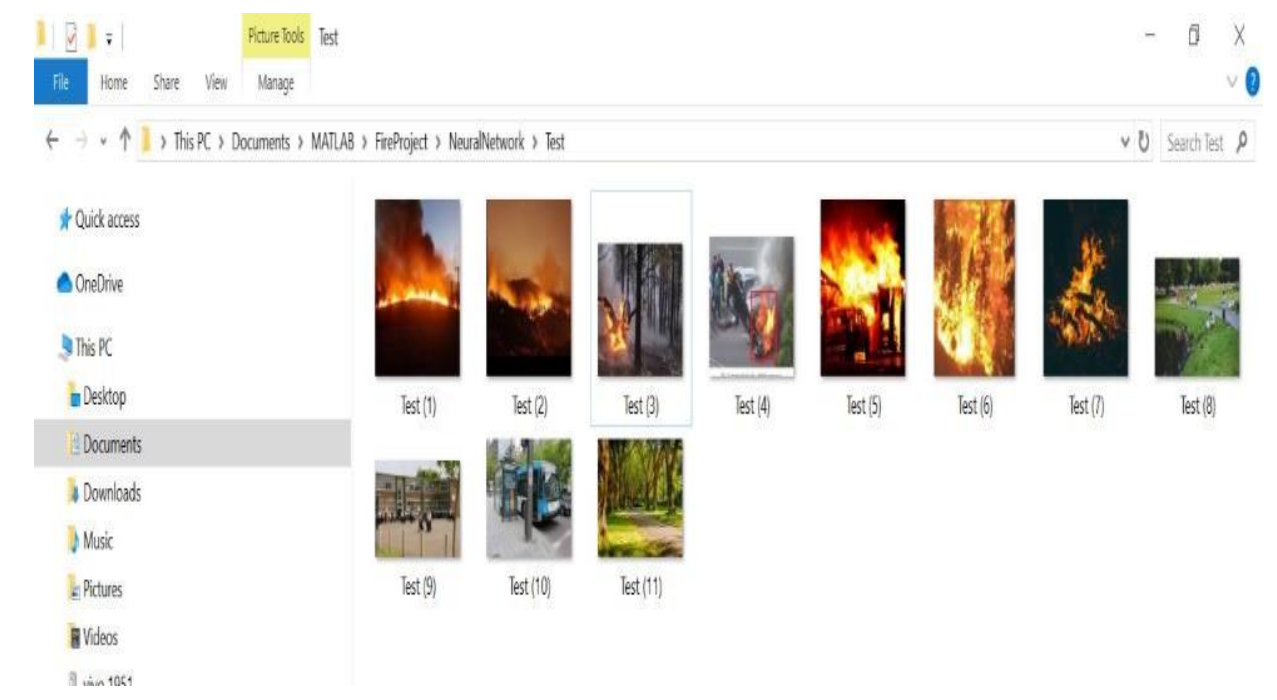

Fig 16 Number of test input

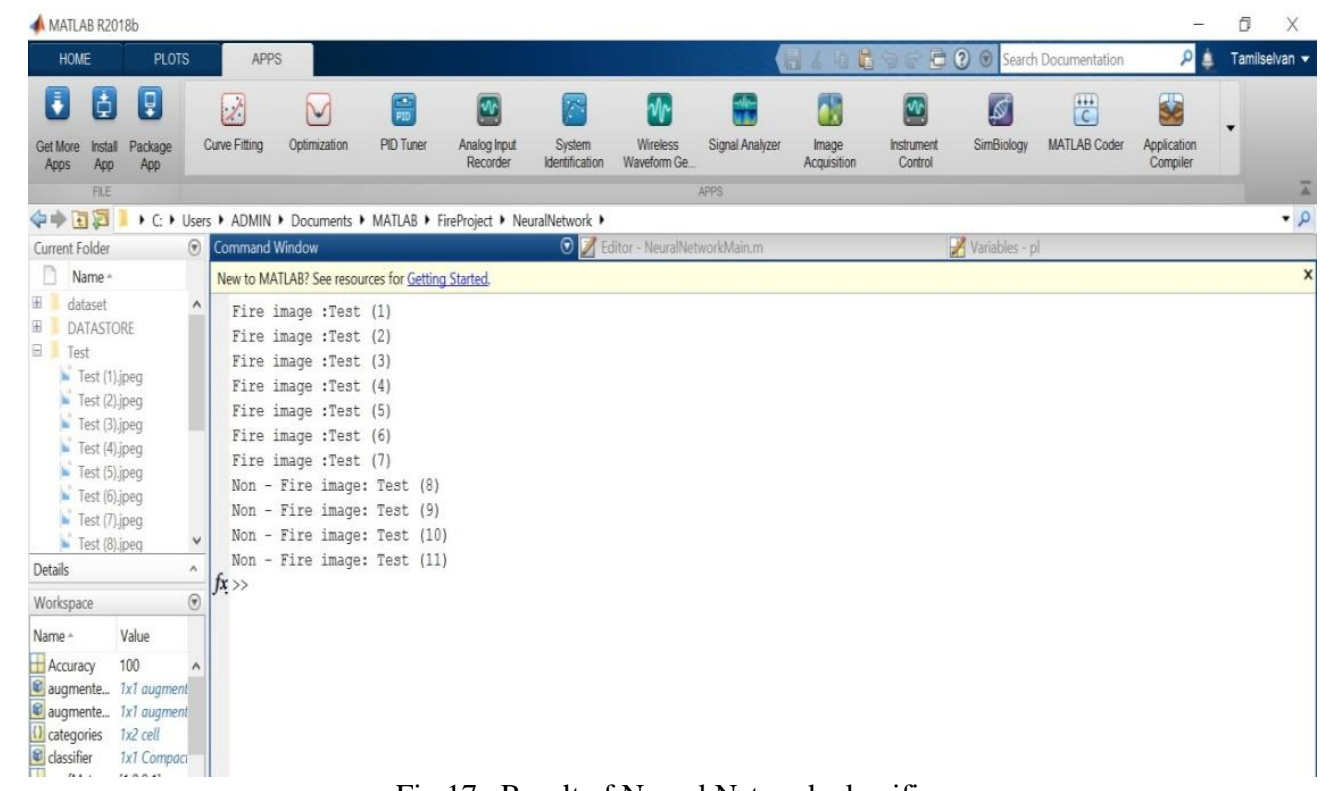

Fig 17 Result of Neural Network classifier 


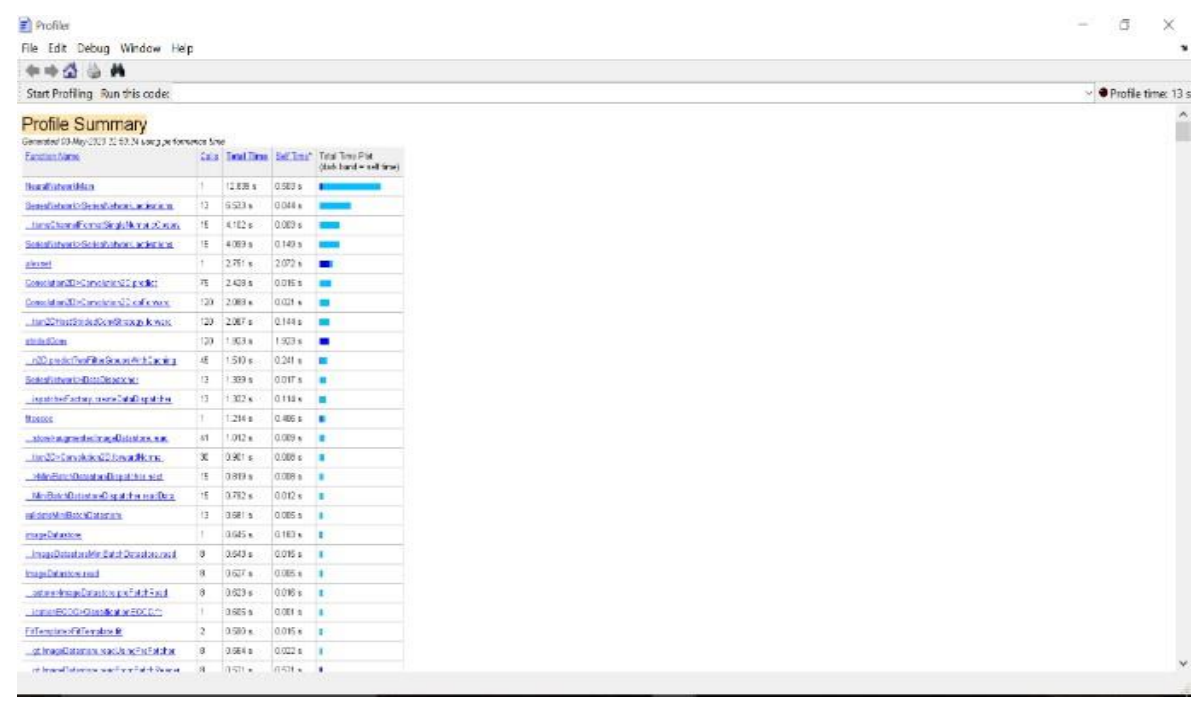

Fig 18 Time Taken for implementation

\section{CONCLUSION}

The accuracy of SVM is $86 \%$ and accuracy of CNN is given by $96 \%$. It is compare to SVM $10 \%$ higher. So we conclude that reliability on image processing to detect fire is increased not only that it lead to overcome the false alarm system.

\section{REFERENCES}

[1] G. Ea J. Yuan and S. Shi-Fei, "On fire hazard indicators and eval- uation modes in public gathering places based on the fire statistical records," Journal of Safety \& Environment13.1, vol. 13, no. 1, pp. 250-253, 2013.

[2] T. Toulouse, X. Maldague, L. Rossi, M.Akhloufi, and T. Celik, "Benchmarking of wildland fire colour segmentation algorithms,” IET. Image Processing, vol. 9, no. 12, pp. 1064-1072, 2015.

[3] T. Chen, P. Wu, and Y. Chiou, "An Early Fire-Detection Method Based on Image Processing," Proc. IEEE Int. Image Process., 2004, pp. 1707-1710.

[4] Poobalan, Kumarguru \& Liew, Siau-chuin. Fire Detection Algorithm Using Image Processing Techniques.Proceeding of the 3rd International Conference on Artificial Intelligence and Computer Science (AICS2015), 12 - 13 October 2015. Penang. Malasiya

[5] Toulouse, Tom \& Rossi, Lucile \& Celik, Turgay \& Akhloufi, Moulay. (2015). Automatic fire pixel detection using image processing: A comparative analysis of Rule-based and Machine Learning-based methods. Signal, Image and Video Processing. June 2015.

[6] Mohanaiah P, Sathyanarayana P, GuruKumar L.Image Texture Feature Extraction Using GLCM Approach. International Journal of Scientific and Research Publication. Volume 3 .Issue 5.May 2013.

[7] T. Toulouse, X. Maldague, L. Rossi, M.Akhloufi, and T. Celik, "Benchmarking of wildland fire colour segmentation algorithms,” IET. Image Processing, vol. 9, no. 12, pp. 1064-1072, 2015.

[8] https://en.wikipedia.org/wiki/Co-occurence_matrix 